\title{
An alarm method for a Loose Parts Monitoring System
}

\author{
Yanlong Cao*, Yuanfeng He, Huawen Zheng and Jiangxin Yang \\ Institute of Manufacturing Engineering, Zhejiang University, Hangzhou, Zhejiang, China
}

Received 17 July 2011

Revised 25 October 2011

\begin{abstract}
In order to reduce the false alarm rate and missed detection rate of a Loose Parts Monitoring System (LPMS) for Nuclear Power Plants, a new hybrid method combining Linear Predictive Coding (LPC) and Support Vector Machine (SVM) together to discriminate the loose part signal is proposed. The alarm process is divided into two stages. The first stage is to detect the weak burst signal for reducing the missed detection rate. Signal is whitened to improve the SNR, and then the weak burst signal can be detected by checking the short-term Root Mean Square (RMS) of the whitened signal. The second stage is to identify the detected burst signal for reducing the false alarm rate. Taking the signal's LPC coefficients as its characteristics, SVM is then utilized to determine whether the signal is generated by the impact of a loose part. The experiment shows that whitening the signal in the first stage can detect a loose part burst signal even at very low SNR and thusly can significantly reduce the rate of missed detection. In the second alarm stage, the loose parts' burst signal can be distinguished from pulse disturbance by using SVM. Even when the SNR is $-15 \mathrm{~dB}$, the system can still achieve a $100 \%$ recognition rate.
\end{abstract}

Keywords: Alarm signal, Loose Parts Monitoring System, Support Vector Machine, Linear Predictive Coding

\section{Introduction}

Loose Parts Monitoring System (LPMS) is one of the basic security tools for Nuclear Power Plants (NPP). The main purpose of LPMS is to detect the loose metallic parts in the Reactor Cooling System (RCS) at an early stage. Several years operating experience in NPP shows that early detection can provide the time required to avoid or mitigate safety-related damage or malfunctions of primary system components [1]. LPMS can also minimize radiation exposure to station personnel.

The main problem of the LPMS is how to detect the small loose parts in a heavy noisy background and discriminate the signal induced by the impact of a loose part from those signals induced by normal hydraulic, mechanical, electrical background noise and amplitude electrical transients.

The traditional method is to compare the amplitude or short-term Root Mean Square (RMS) of a signal to a given threshold. Once the threshold is exceed, a discrimination program is triggered immediately to identify the real signal caused by loose parts. At present, research is focused on how to identify whether the signal is induced by a loose part. Owing to that the impact signal can be detected by more than one sensor and single sensor can be much easier influenced by the occasional interference signal, Morel and Puyal [2] proposed defining different thresholds for sensors installed on different position of the plant to compensate the background noises and then checking if more than one channel signals' amplitudes exceed the threshold so as to determine whether to trigger the alarm. Due to the property that the time-varying of the metal impact signal's frequency spectrum is obvious while

${ }^{*}$ Corresponding author: Dr. Cao Yanlong, Institute of Manufacturing Engineering, Zhejiang University, 38 Zheda Road, Hangzhou 310027, China. Tel.: +86 571 87953198; E-mail: sdcaoyl@zju.edu.cn. 


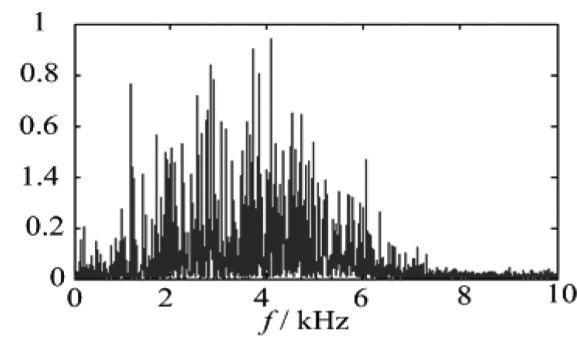

(a) Spectrum of impact signal

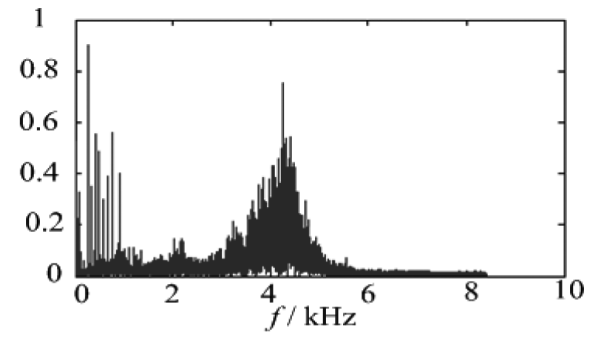

(b)Spectrum of noise signal

Fig. 1. The spectrum of burst signal and noise signal.

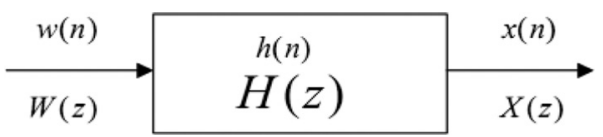

Fig. 2. Parametric model of stochastic signal.

that of the background noise is relatively stable, Mao [3] proposed expressing the impact signal feature using the two-dimension characteristic in the signal's time-frequency domain and using BP neural network as impact signal classifier to determine if the signal is a real alarm. Since the complexity of the impact signals' various properties, Lenain [4] defined a three level alarm criteria including defining a correlation graph of two signal feature to generate the suspect alarm, performing pattern recognition analysis to start the alarm determination process and analyzing the frequency spectrum content of the signal, the alarm is triggered only after all the three conditions are satisfied so as to ensure the reliability of loose part alarm. Utilizing the difference of the impact signal and interference signal in amplitude and waveform, Figedy [5] proposed decomposing the signal using discrete wavelet transform with Haar wavelet basis, considering that the eighth level approximation coefficients as the expression of the signal feature and the artificial neural network is then used to determine if the signal is a false alarm according to the given feature. Tjhai [6] developed a two-stage classification system using the combination of two data mining techniques, namely SOM(Self-Organizing Map) and K-means clustering. First stage was developed to properly correlate alerts related to a particular activity. The main objective of the second stage is to subsequently label all the clusters produced in the first-stage classification into groups of true and false alarms. Based on the existence of time difference of detecting the vibration signals generated by the loose parts by sensors amounted on different positions, Zigler [7] proposed judging whether the suspect signal is impact signal or not according to the compatibility of signal detecting time among the related sensors.

According to the difference between the spectrum characteristics of loose part impact signals and other pulse disturbances, an alarm method which takes LPC of the signals as the signal feature and uses SVM to distinguish the impact signals is proposed. The method of detecting the burst signal is illustrated in Section 2. The theory of extracting the feature of signals and distinguishing the signal by SVM are stated in Sections 3 and 4, respectively. Section 5 shows the experiment and the results of data analysis. The paper is concluded in Section 6.

\section{Signal detection}

As Fig. 1 shows, the burst signal spectrum is similar to the noise spectrum. When the SNR is low, the burst signal could not be detected. Thus, adaptive AR model is used as a signal preprocessing method to eliminate the influence of background noise.

The RCP's background noise $x(n)$ can be regarded as a system response to the white noise $w(n)$.

Using the AR model to describe this system, the system transfer function can be written as:

$$
H(z)=\frac{1}{1+\sum_{k=1}^{p} a_{k} z^{-k}}
$$




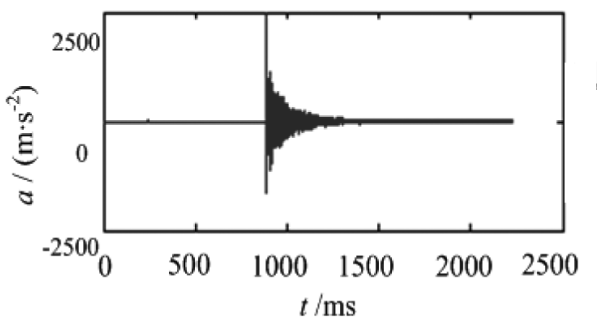

(a) Impact signal

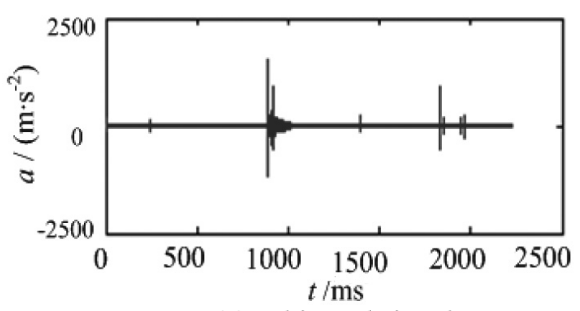

(c) Whitened signal

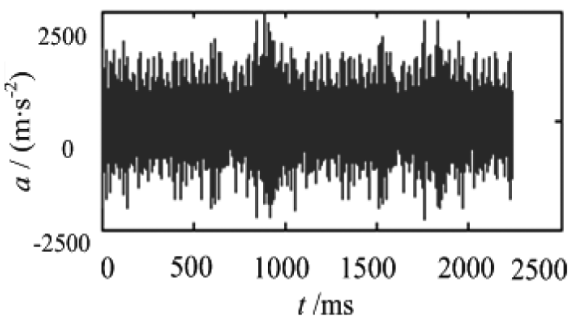

(b) Mixed signal

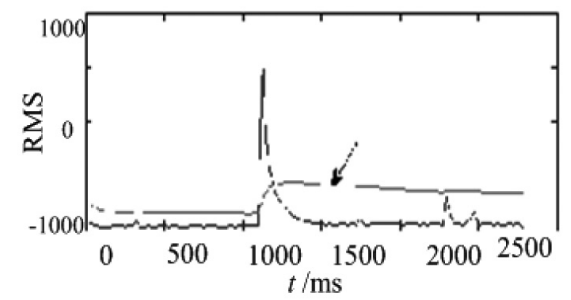

(d) Short-term RMS of whitened signal

Fig. 3. Signal whitening.

where $p$ is the order of the system; the coefficients $a_{k}$ can be estimated by an efficient algorithm known as the Levinson-Durbin algorithm from a given background noise $x(n)$. A whitening filter whose transfer function is $\frac{1}{H(z)}$ can then be acquired. Thus, given the noise $x(n)$, one can obtain the white noise $w(n)$ :

$$
Y=\left\{y_{1}, y_{2}, \cdots, y_{h}\right\}
$$

When a loose part comes out, the recorded signal consists of a burst signal and a background noise signal. Generally, the frequency range of noise is from $3 \mathrm{kHz}$ to $5 \mathrm{kHz}$ while that of burst signal is from $1 \mathrm{kHz}$ to $10 \mathrm{kHz}$. After the recorded signal whitened, the background noise becomes Gaussian White noise, but the burst signal cannot be completely whitened. Therefore, the signal's SNR can be greatly improved.

In Fig. 3, (a) is the loose part impact signal, (b) is the recorded signal, which consists of a burst signal and a noise signal, and the SNR is $-5 \mathrm{~dB}$, (c) is the whitened signal, and (d) is the whitened signal's short-time RMS. From Fig. 3 we can find out that the whitening filter can improve the SNR substantially. The burst signal can be detected easily by check the whitened signal's short-time RMS.

There are two types of interference that can easily induce false alarming. One is the burst signal caused by a control rod movement. This kind of signal is similar to the burst signal caused by loose parts. Another type of interference is electromagnetic interference, which can also lead to false alarming. There are many sources of electromagnetic interference, including the reactor's complex background noise, radiation from the interference, and lightning.

Wik [8] expressed the Nuclear Electromagnetic Pulse (NEMP), Static Electromagnetic Pulse (ESDEMP), and Lightning Electromagnetic Pulse (LEMP) in a double-exponential decay pulse signal model. This model was used as an interference pulse generation model in this paper. Its waveform is:

$$
s(t)=A\left(e^{-t / \tau_{1}}-e^{-t / \tau_{2}}\right)
$$

Given the different value, $\tau_{1}$ and $\tau_{2}$ can generate the pulses of different shapes.

The generated pulse and background noise are mixed together to prepare for whitening, the short-time RMS is calculated as shown in Fig. 5.

From Fig. 5 we can see that the whitening filter cannot eliminate the pulse's interference, and the LPMS would raise a false alarm by checking the short-term RMS of a whitened signal. Therefore, a further analysis should be undertaken to distinguish between the interference and burst signal. 


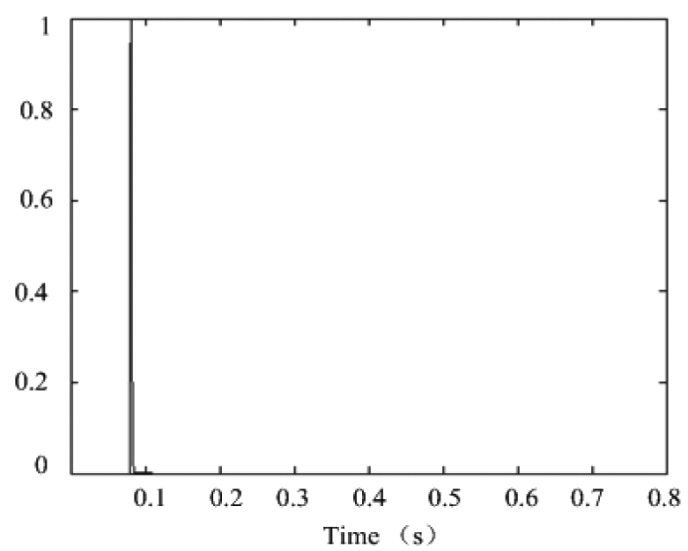

(a) Generated pulse with $\tau_{1}=1 \mathrm{~ms} \tau_{2}=0.5 \mathrm{~ms}$

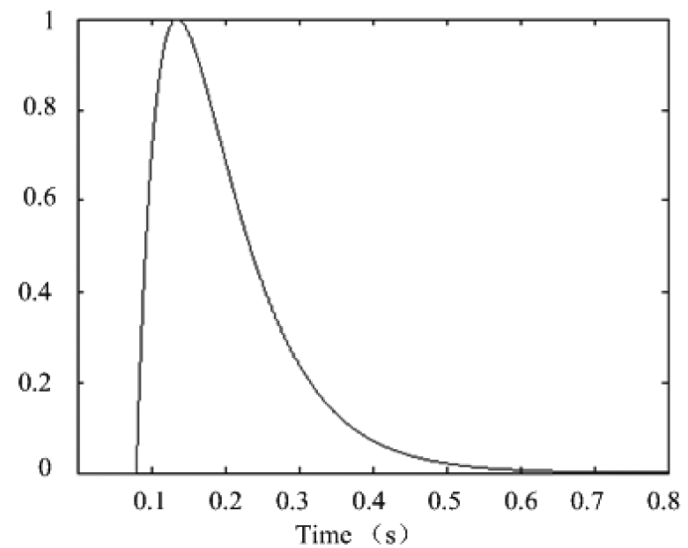

(b) Generated pulse with $\tau_{1}=100 \mathrm{~ms} \tau_{2}=50 \mathrm{~ms}$

Fig. 4. Generated impulse.
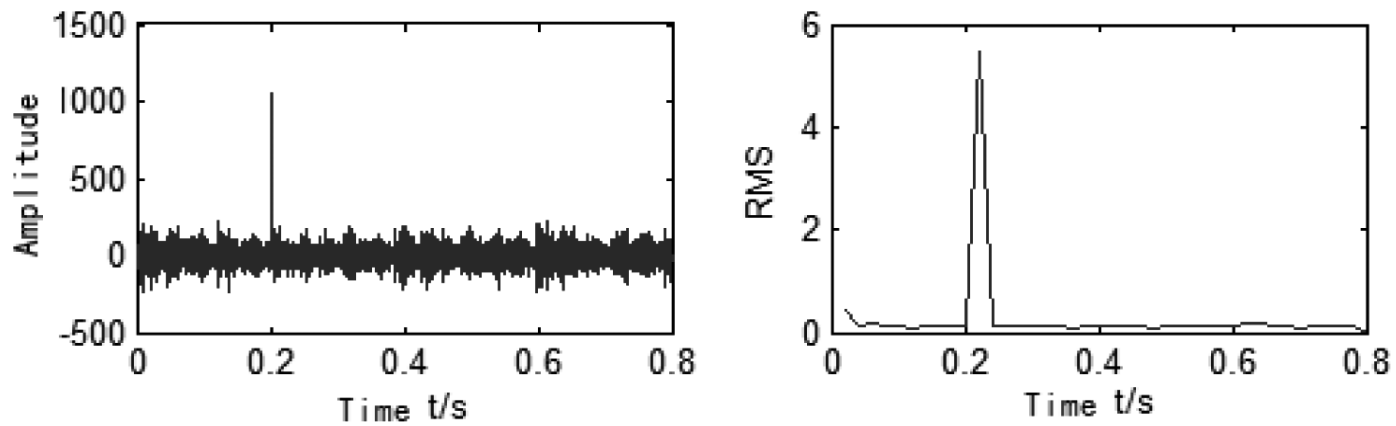

Fig. 5. Process result of signal with impulse.

\section{Signal feature extraction}

The recorded signal should be analyzed to determine whether it is a false detection after it has been detected. The most important step of all recognition systems is the signal feature extraction, which converts the signal waveform to some type of parametric representation. This parametric representation is then used for further analysis.

Loose part's impact signal and pulse with noise is mixed with different SNR $(10 \mathrm{~dB}$ and $-10 \mathrm{~dB})$, and then whitened. The waveform and spectrum of the whitened signal is shown in Figs 6 and 7.

Figures 6 and 7 show that the whitened impact signal waveform and pulse signal waveform are similar when the SNR is $-10 \mathrm{~dB}$, but the spectrum of the whitened impact signal and the pulse signal are different. Thus, the impact signal can be identified based on the signal spectrum information.

Since the sampling rate is high, the signal has large amounts of data that will result in very time-consuming calculation, and there is huge redundancy within the data. Thus, one should collect the characteristics that reflect the nature of the signal in order to compress the data. In this paper, linear predictive coding (LPC) coefficients are utilized to express the signal spectrum. The AR model parameters $a_{k}$ in formula Eq. (1) is now the LPC coefficients. As shown in Fig. 7, signal's spectrum (red) estimated using LPC can be a good fit of its FFT spectrum (blue).

\section{Support Vector Machine}

A stable efficient classification algorithm is needed to identify the loose part impact signal after the signal feature is extracted. SVM is chosen to be the classifier in this paper. SVM is a statistical classification method proposed by 


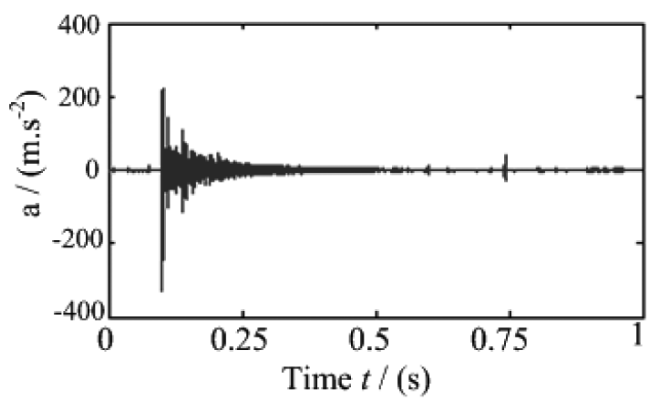

(a) Whitened impact signal

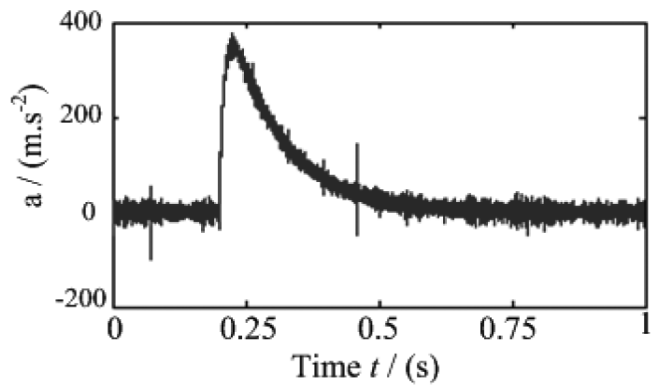

(c) Whitened pulse

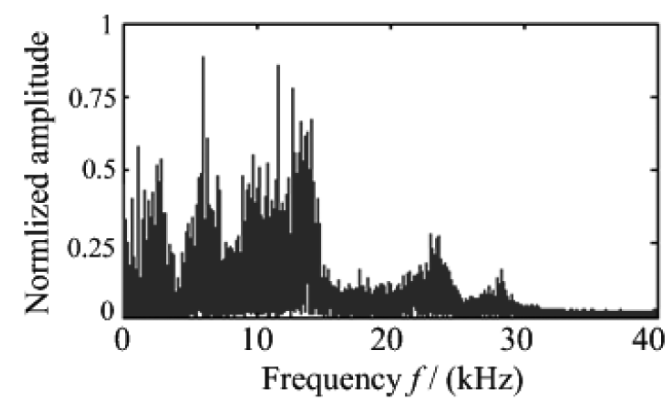

(b) Spectrum of whitened impact signal

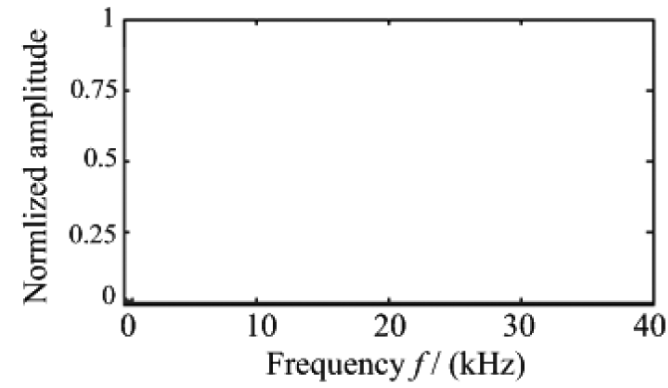

(d) Spectrum of whitened pulse

Fig. 6. Pulse signal with noise and its normalized spectrum after whitening (10 dB).

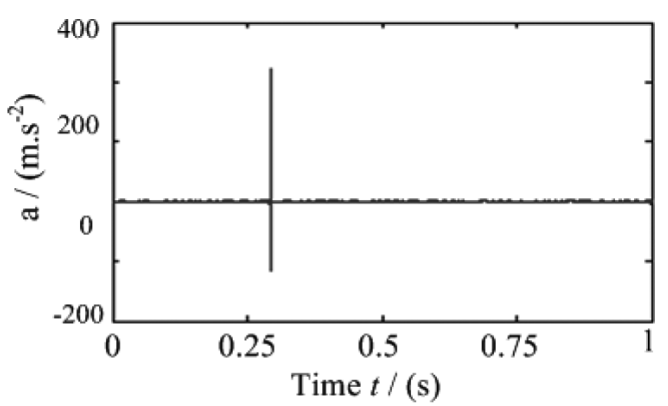

(a) Whitened impact signal

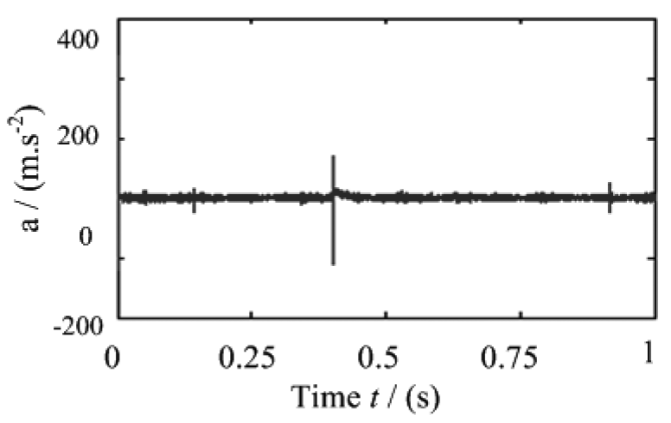

(c) Whitened pulse

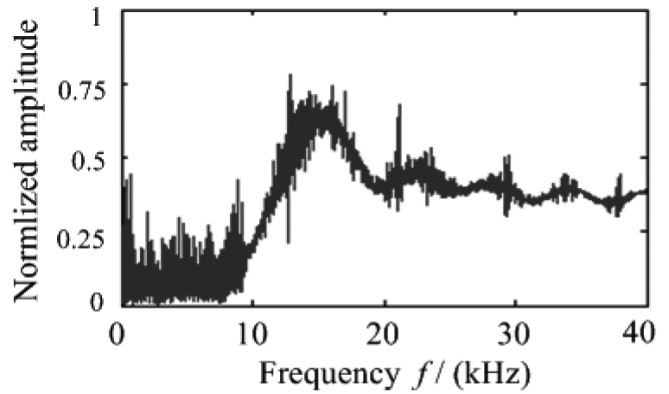

(b) Spectrum of whitened impact signal

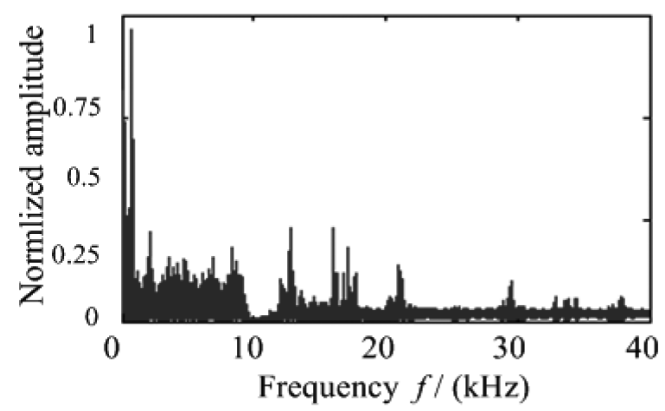

(d) Spectrum of whitened pulse

Fig. 7. Pulse signal with noise and its normalized spectrum after whitening $(-10 \mathrm{~dB})$. 


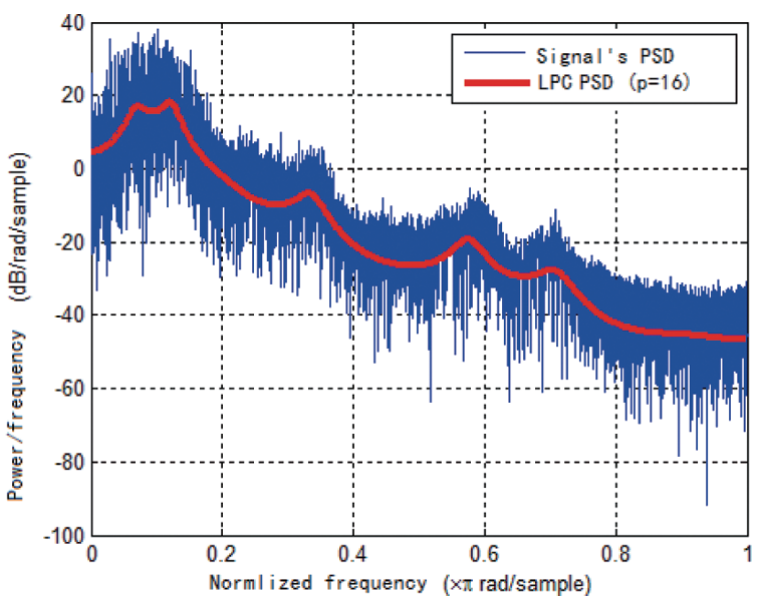

Fig. 8. Signal FFT spectrum and LPC estimated spectrum.

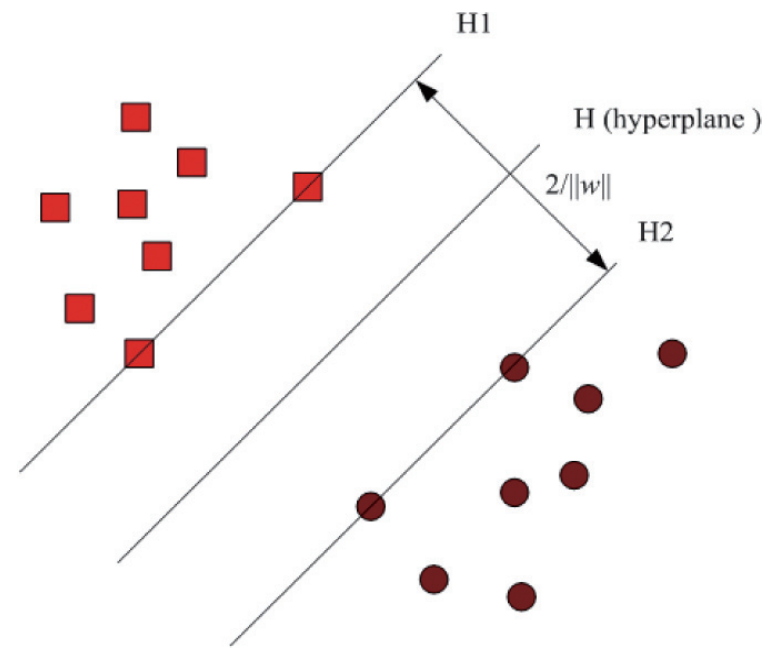

Fig. 9. SVM optimal hyperplane.

Vapnik [9], developed on the basis of statistical learning theory with good classification ability and generalization ability. The basic idea is to define the best optimal hyperplane, which separates sample points into two categories and makes the distance from the training samples to the hyperplane as far apart as possible.

Given the training data $\left(x_{k}, y_{k}\right), k=\{1, \cdots, h\}, x_{k} \in R^{m}, y_{k} \in\{1,-1\}$, the separating hyperplane can be described by the equation: $g(x)=\left\langle\omega, x_{k}\right\rangle+b=0$. Let the closest point to the hyperplane has a distance of $1 /\|\omega\|$ to rescale the $\omega$ and $b$. Thus:

$$
y_{k}\left[\left\langle\omega, x_{k}\right\rangle+b\right]-1 \geqslant 0
$$

The quantity $2 /\|\omega\|$ is called the margin. Computing the hyperplane is equivalent to minimize $\|w\|^{2}$. Since $\|w\|^{2}$ is convex, minimizing it under linear constraints can be achieved with Lagrange multipliers. The optimization problem can be presented as a dual problem:

$$
\begin{aligned}
& \max Q(\alpha)=\sum_{i=1}^{H} \alpha_{i}-\frac{1}{2} \sum_{i, j=1}^{H} \alpha_{i} \alpha_{i} y_{i} y_{j}\left\langle x_{i}, x_{j}\right\rangle \\
& \text { s.t. }\left\{\begin{array}{l}
C \geqslant \alpha_{i} \geqslant 0 \\
\sum_{i=1}^{H} y_{i} \alpha_{i}=0
\end{array}\right.
\end{aligned}
$$

The support vectors are the points for which $\alpha_{i}^{*}>0$ satisfy Eq. (4) with equality. Then we can find $b^{*}$. The hyperplane decision function can thus be written as:

$$
g(x)=\operatorname{sgn}\left(\sum_{i=1}^{H} \alpha_{i}^{*} y_{i}\left\langle x_{i}, x\right\rangle+b^{*}\right)
$$

When the data is not linearly separable, the kernel function is introduced to map the input data into a highdimensional feature space. A typical kernel is the RBF (Radial Basis Function) kernel:

$$
K\left(x_{i}, x\right)=\exp \left(-\frac{\left|x-x_{i}\right|^{2}}{\sigma^{2}}\right)
$$

Once the kernel has been chosen, the decision function becomes:

$$
g(x)=\operatorname{sgn}\left(\sum_{i=1}^{H} \alpha_{i}^{*} y_{i} K\left(x_{i}, x\right)+b^{*}\right)
$$

The decision function has a complexity that increases with training set size. Moreover, high dimensional spaces are sensitive to the curse of dimensionality and scalar products quickly become difficult to compute. Thus, we use the 
Table 1

The missed detection rate $(\%)$ when the LPCs is 16

\begin{tabular}{lccrrrrrr}
\hline & \multicolumn{10}{c}{ SNR } \\
\cline { 2 - 9 } & $20 \mathrm{~dB}$ & $10 \mathrm{~dB}$ & $0 \mathrm{~dB}$ & $-5 \mathrm{~dB}$ & $-10 \mathrm{~dB}$ & $-15 \mathrm{~dB}$ & $-20 \mathrm{~dB}$ & $-30 \mathrm{~dB}$ \\
\hline Signal not whitened & 6.7 & 6.7 & 45.0 & 68.3 & 76.7 & 100.0 & 100.0 & 100.0 \\
Signal whitened & 0.0 & 0.0 & 0.0 & 0.0 & 11.7 & 31.7 & 31.7 & 23.3 \\
\hline
\end{tabular}

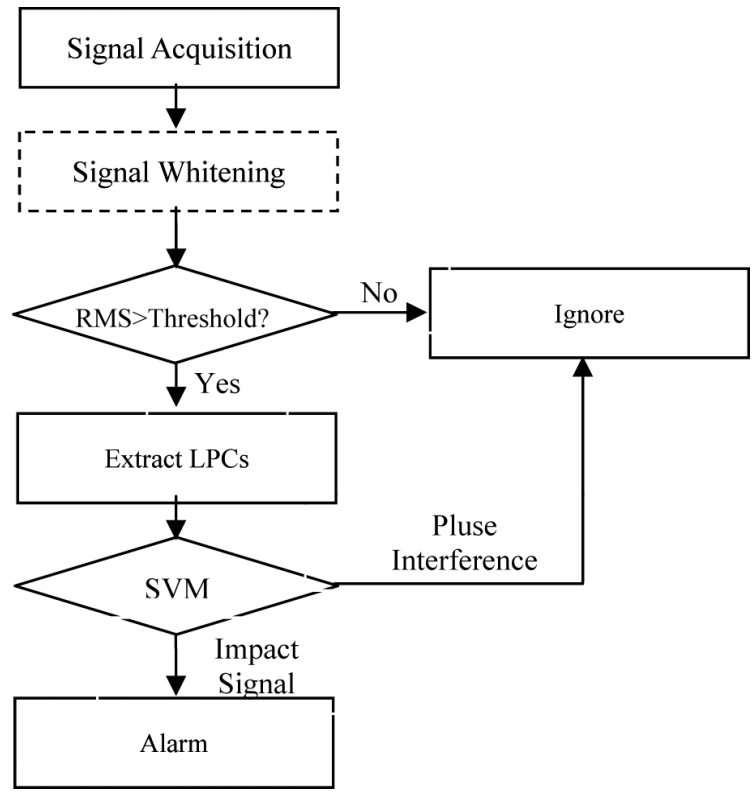

Fig. 10. System alarm process

LPC coefficients of signal as the input data, which is dimensionality reduced compared with the original spectrum. The reduction can ease the learning process and improve generalization abilities.

\section{Experimental verification}

The sample signals are from testing with different weight steel ball $(10 \mathrm{~g}, 30 \mathrm{~g}, 55 \mathrm{~g}, 110 \mathrm{~g}, 185 \mathrm{~g}$, and $720 \mathrm{~g})$ to hit the steel plate, and the signal's sampling rate is $80 \mathrm{kHz}$. The interference pulse signal can be calculated from formula Eq. (4) with different widths and shapes by setting $\tau_{1}$ and $\tau_{2}$ different value.

The system alarm process is shown as Fig. 10. In order to simulate the real signal environment, the collision signal and pulse signal are superposed with the reactor noise (collected from the Qingshan Nuclear Power Plant in China) by different SNR. Then these signals are whitened and then compared its short-term RMS to the threshold. If it exceeds the threshold, then the signal's feature is extracted and input into the trained SVM model to determine whether the detected signal is an impact signal.

The threshold value can be calculated as:

$$
T_{h}=\lambda \times R M S_{\text {background }}
$$

where $T_{h}$ is threshold value $£<\lambda$ is coefficient $£$ <reduce $\lambda$ can reduce miss alarm rate at some extent, $R M S_{\text {background }}$ is long-term RMS of background noise.

There are 30 impact signals and 30 pulse signals for training samples, and the SNR is $0 \mathrm{db}$. There are 60 impact signals and 60 pulse signals as the test samples, using different SNRs to superimpose the nuclear power plant background noise. We use training samples to train the SVM firstly and input the test samples into the experiment then to get the results shown in Tables 1,2 and 3. 
Table 2

The false alarm rate(\%) when the LPCs is 16

\begin{tabular}{lcccccccc}
\hline & \multicolumn{7}{c}{$\mathrm{SNR}$} \\
\cline { 2 - 9 } & $20 \mathrm{~dB}$ & $10 \mathrm{~dB}$ & $0 \mathrm{~dB}$ & $-5 \mathrm{~dB}$ & $-10 \mathrm{~dB}$ & $-15 \mathrm{~dB}$ & $-20 \mathrm{~dB}$ & $-30 \mathrm{~dB}$ \\
\hline Signal not whitened & 0.0 & 0.0 & 0.0 & 0.0 & 0.0 & 0.0 & 0.0 & 0.0 \\
Signal whitened & 0.0 & 0.0 & 0.0 & 0.0 & 0.0 & 0.0 & 0.0 & 71.7 \\
\hline
\end{tabular}

Table 3

The system recognition $\operatorname{rate}(\%)$

\begin{tabular}{crrrrrrrc}
\hline LPC Number & \multicolumn{7}{c}{ SNR Test sample } \\
\cline { 2 - 8 } & $20 \mathrm{db}$ & \multicolumn{1}{c}{$10 \mathrm{db}$} & \multicolumn{1}{c}{$0 \mathrm{db}$} & $-5 \mathrm{db}$ & $-10 \mathrm{db}$ & $-15 \mathrm{db}$ & $-20 \mathrm{db}$ & $-30 \mathrm{db}$ \\
\hline 1 & 94.17 & 95.83 & 100.00 & 100.00 & 100.00 & 99.17 & 89.17 & 60.00 \\
2 & 100.00 & 100.00 & 100.00 & 100.00 & 100.00 & 100.00 & 97.50 & 65.00 \\
4 & 100.00 & 99.17 & 96.67 & 94.17 & 89.17 & 85.00 & 84.17 & 87.50 \\
8 & 100.00 & 100.00 & 100.00 & 100.00 & 94.17 & 84.17 & 84.17 & 88.33 \\
16 & 100.00 & 100.00 & 100.00 & 100.00 & 94.17 & 85.00 & 84.17 & 88.33 \\
32 & 100.00 & 100.00 & 100.00 & 100.00 & 90.00 & 85.00 & 60.00 & 39.17 \\
64 & 100.00 & 100.00 & 100.00 & 100.00 & 94.17 & 85.83 & 43.33 & 40.00 \\
128 & 100.00 & 100.00 & 100.00 & 100.00 & 94.17 & 86.67 & 43.33 & 40.00 \\
256 & 100.00 & 100.00 & 100.00 & 100.00 & 94.17 & 87.50 & 42.50 & 40.83 \\
\hline
\end{tabular}

As Table 1 shows, the missed detection rate increases when the SNR becomes lower. The whitening preprocess can reduce the missed detection rate significantly. Table 2 shows that SVM can identify the pulse interference when the SNR is very low.

Table 3 shows the system recognition rate when the number of LPCs is different. It happens to have the best recognition result when the number of LPCs is 2 . And if the SNR is $-15 \mathrm{db}$, this provides a $100 \%$ recognition rate. There is still a $97.5 \%$ recognition rate even when the SNR is down to $-20 \mathrm{db}$.

\section{Conclusion}

This paper presents an alarm method of LPMS based on the AR model and SVM. The system alarm is divided into two stages for a low false alarm rate and low missed alarm rate. By experimental verification, we have reached the following conclusions: (1) the whitening process of the background noise based on AR modeling can effectively improve the SNR. It can reduce the missed detection rate; (2) extracting LPCs of signal as the input data of SVM can reduce the dimension of data, and when the number of LPC parameter is 2 the best recognition results occur. As long as the SNR is greater than $-15 \mathrm{db}$, the false alarm rate and missed alarm rate are reduced to zero.

\section{Acknowledgments}

This study was supported by a grant from the National Natural Science Foundation of the P. R. China (No. 51175466 ) and Qianjiang Talents Project of Science Technology Department of Zhejiang, China (No. 2011R10016).

\section{References}

[1] B. Bechtold and U. Kunz, KUES'95-The modern diagnostic system for loose parts monitoring [J], Progress in Nuclear Energy 34(3) (1999), 221-230.

[2] J.L. Morel and C. Puyal, On-line acoustic monitoring of EDF nuclear plants in operation and loose-part diagnostics, SMORN VI, Gatlinburg, Tennessee USA, 1991.

[3] H.L. Mao, Z.F. Huang and Z.Y. Cheng, Artificial neural network alarm method based on signal time-frequency characteristics [J], Nuclear Power Engineering 19(3) (1998), 265-269 (in Chinese).

[4] J.C. Lenain and M.F. Carlos, Acoustic emission (AE) loose parts monitoring application in a nuclear power plant, EWGAE 2004.

[5] S. Figedy and G. Oksa, Modern methods of signal processing in the loose part monitoring system[J], Progress in Nuclear Energy 46(3-4) (2005), 253-267. 
[6] G.G. Tjhai, S.M. Furnell, M. Papadaki et al., A preliminary two-stage alarm correlation and filtering system using SOM neural network and K-means algorithm, Computers and Security 29 (2010), 712-723.

[7] G.R. Zigler, On-line Loose Parts Monitoring False Alarm Discrimination, Progress in Nuclear Energy 15(3) (1985), $577-581$.

[8] M.W. Wik, Double exponential of optical and electrical signals and pattern recognition in lightning, nuclear and electrostatic discharge spectra [C], Proc of 6th Int Symp Tech Exhibition on Electromagnetic Compatibility (1985), 169.

[9] V. Vapnik, S. Golowich and A. Smola, Support vector method for function approximation, regression estimation, and signal processing, in: Advances in Neural Information Processing Systems 9, M. Mozer, M. Jor-dan and T. Petsche, eds, Cambridge, MA, 1997, pp. $281-287$. MIT Press. 

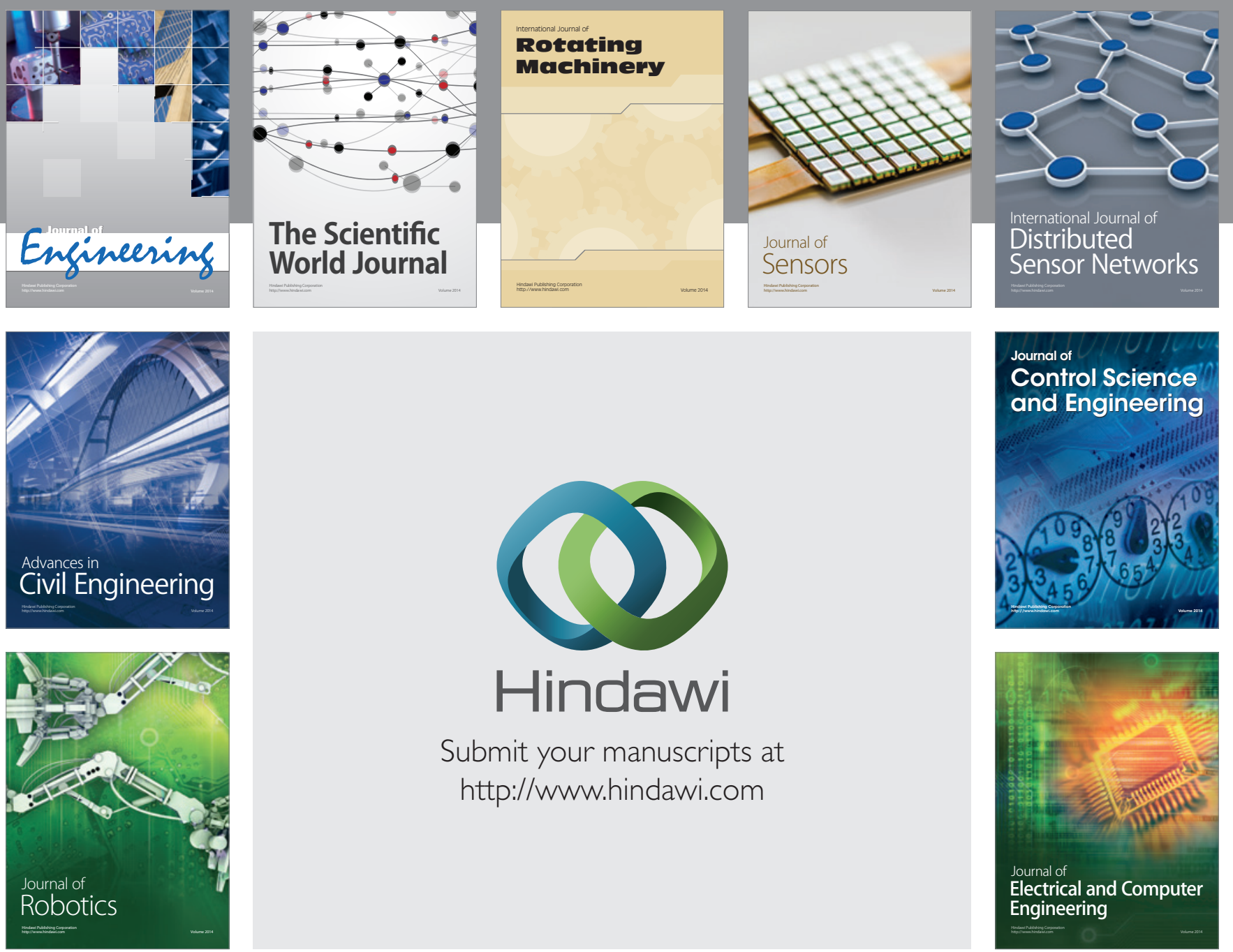

Submit your manuscripts at

http://www.hindawi.com
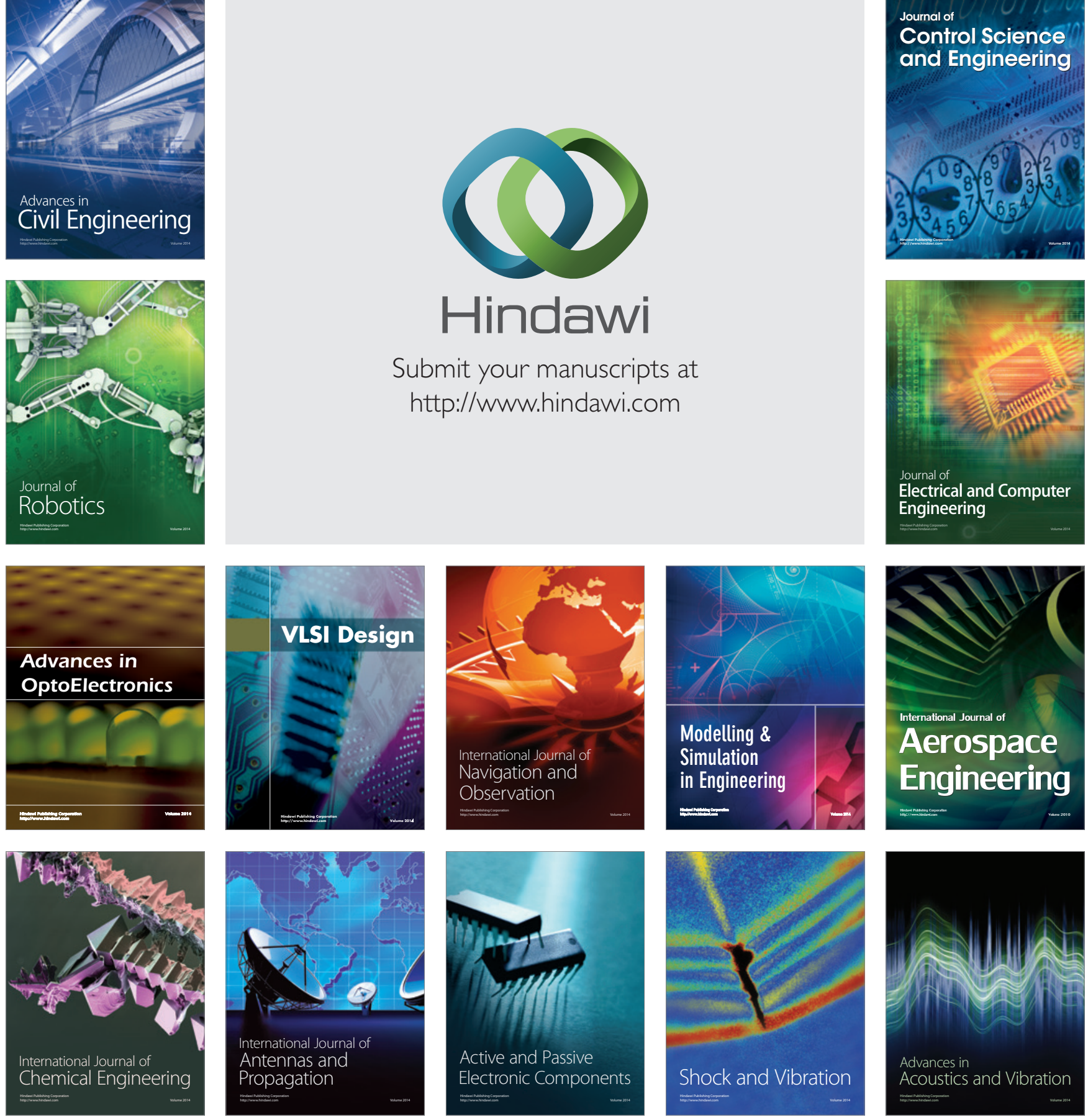